\title{
Comparison of Degradation Rates of Individual Modules Held at Maximum Power
}

\section{C.R. Osterwald, J. Adelstein, J.A. del Cueto, B. Kroposki, D. Trudell, and T. Moriarty}




\section{Disclaimer and Government License}

This work has been authored by Midwest Research Institute (MRI) under Contract No. DE-AC36-99G010337 with the U.S. Department of Energy (the "DOE"). The United States Government (the "Government") retains and the publisher, by accepting the work for publication, acknowledges that the Government retains a non-exclusive, paid-up, irrevocable, worldwide license to publish or reproduce the published form of this work, or allow others to do so, for Government purposes.

Neither MRI, the DOE, the Government, nor any other agency thereof, nor any of their employees, makes any warranty, express or implied, or assumes any liability or responsibility for the accuracy, completeness, or usefulness of any information, apparatus, product, or process disclosed, or represents that its use would not infringe any privately owned rights. Reference herein to any specific commercial product, process, or service by trade name, trademark, manufacturer, or otherwise does not constitute or imply its endorsement, recommendation, or favoring by the Government or any agency thereof. The views and opinions of the authors and/or presenters expressed herein do not necessarily state or reflect those of MRI, the DOE, the Government, or any agency thereof. 


\section{Outline}

- Purpose

- Published degradation rates

- NREL measurement procedure

- Degradation rate results

- Discussion and conclusions 


\section{Purpose}

- Module degradation rates $\left(R_{D}\right)$ needed for accurate PV system energy delivery calculations

- Time-consuming measurement

- $R_{D}$ data are generally unavailable

- System sizing software PVWATTS:

- Has an input for aging loss, but defaults to no loss

- Recommends using the common rule-of-thumb $1 \%$ per year

- Attempt to quantify PV module $R_{D}$ 


\section{Published Degradation Rates}

- PV literature search for published $R_{D}$ values

- Only nine references since 2000 found

- Indication of measurement difficulties

- All but two are from modules exposed in systems

- $R_{D}$ values derived from system data can include factors unrelated to modules, such as:

- Inverter operation; max. power tracking

- Wiring degradation

- System exposures provide more statistics 


\section{Published $R_{D}-$ Systems}

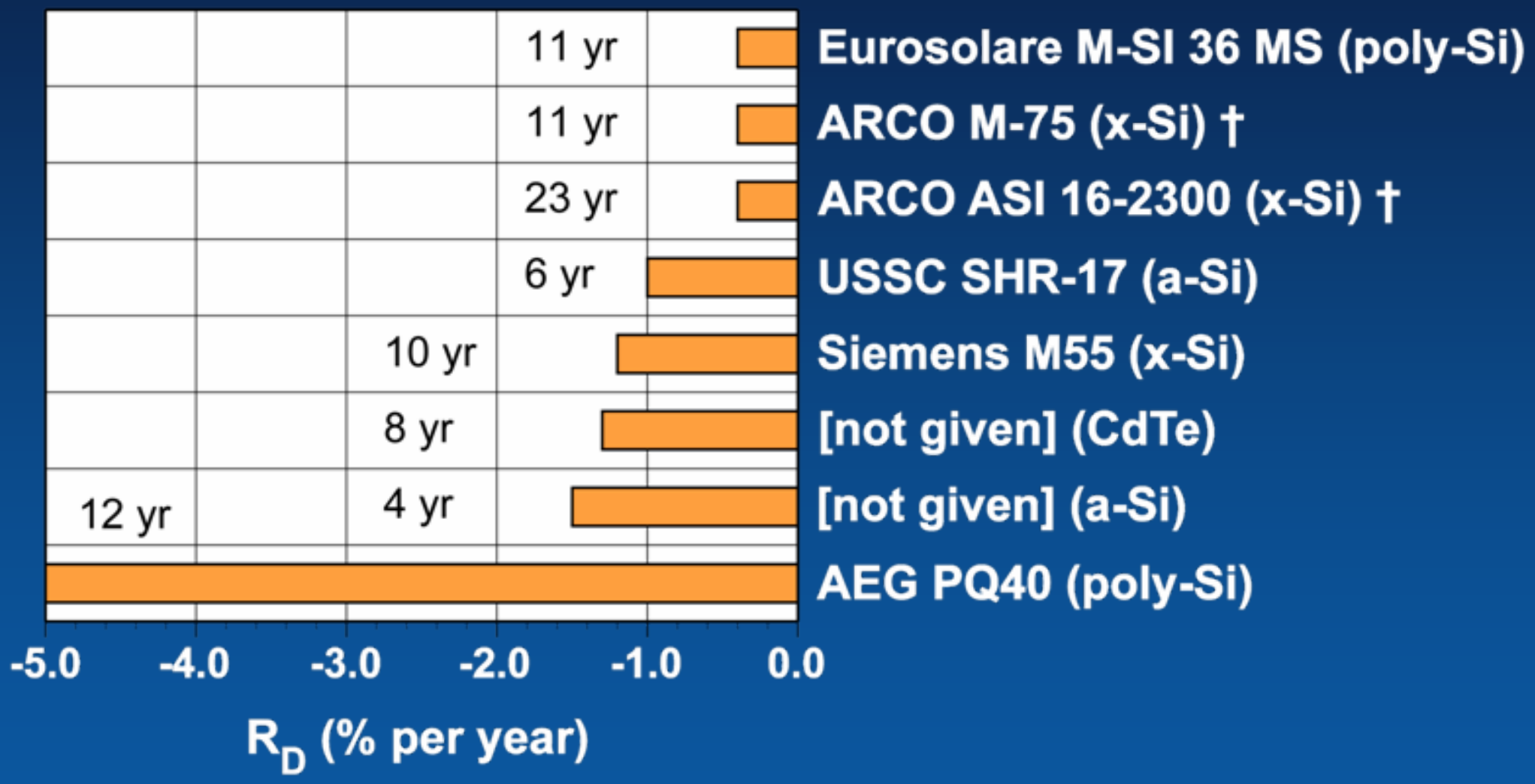

† Based on individual module performance measurements 


\section{Published $\mathbf{R}_{\mathrm{D}}-$ Modules}

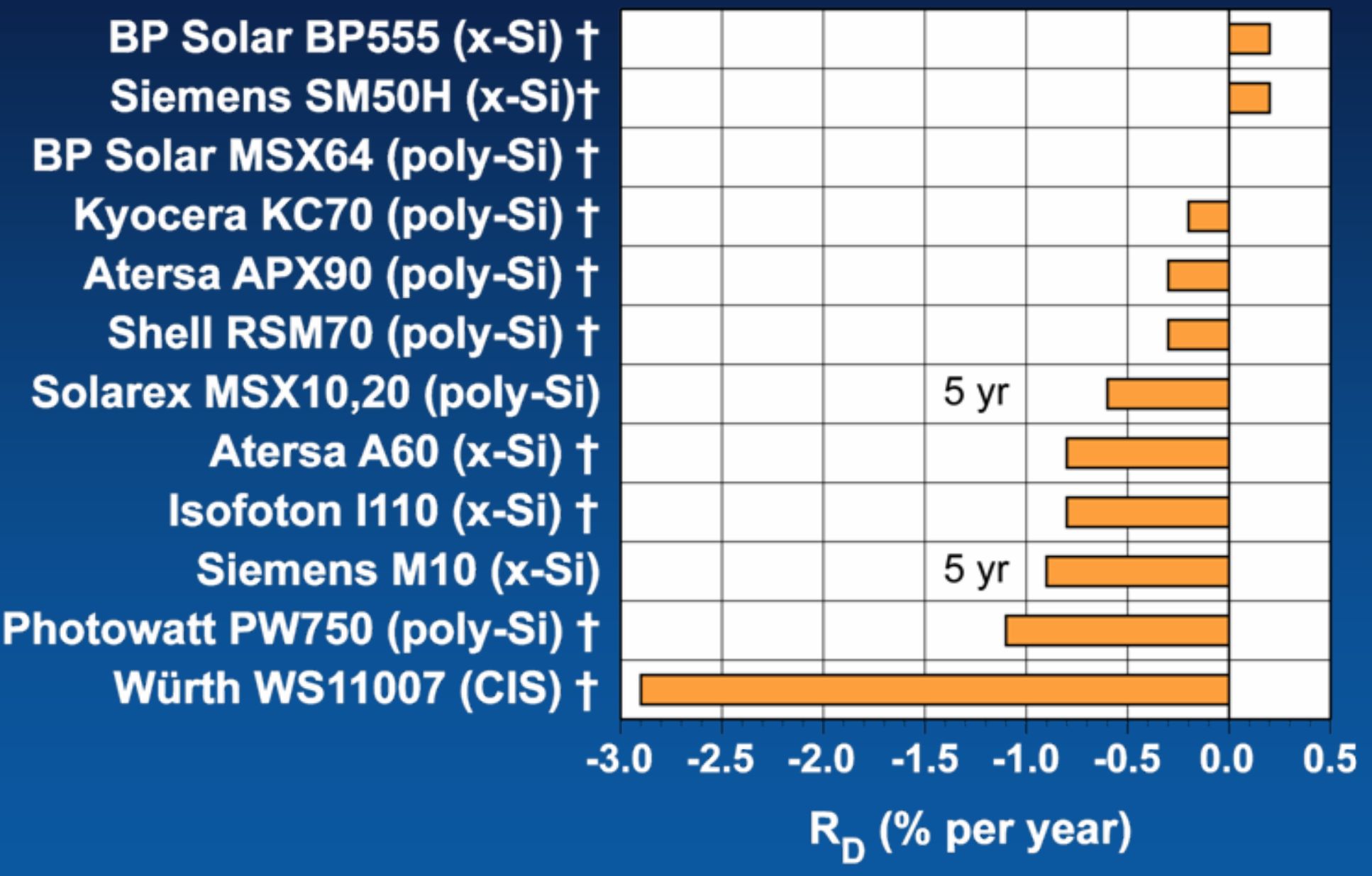

† N. Cereghetti, et. al., 3rd WCPEC, Osaka, May 2003 


\section{NREL $R_{D}$ Measurements}

- Performance \& Energy Ratings Testbed (PERT) on roof of Outdoor Test Facility

- Operational since 1994

- Currently 35 modules under test

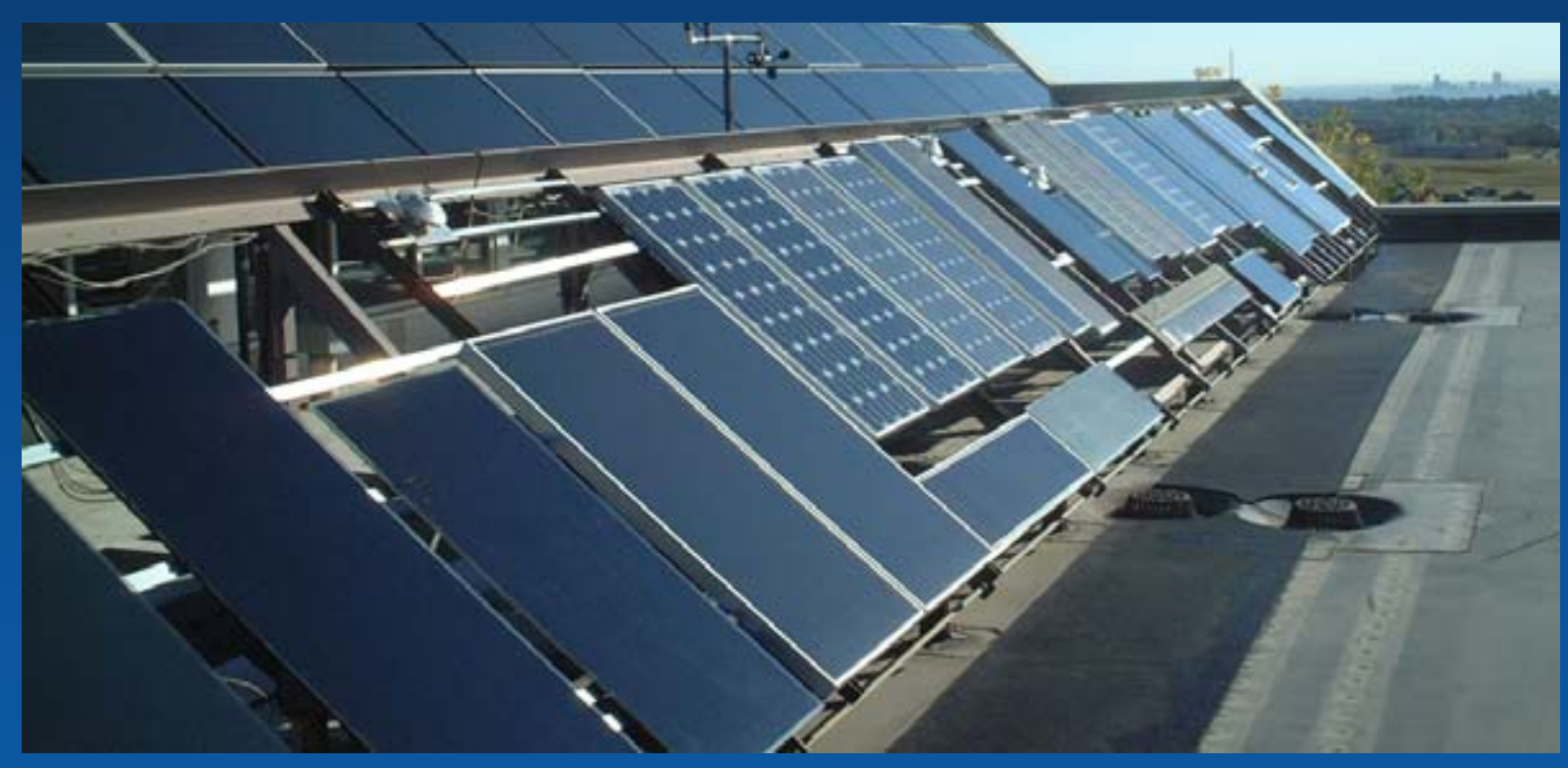




\section{PERT Data Acquisition}

- 3 Raydec Multi-Tracer II max-power tracking loads

- 15 module channels each

- I-V curves every 15 min.

- Irradiance and back-ofmodule temperature measurements

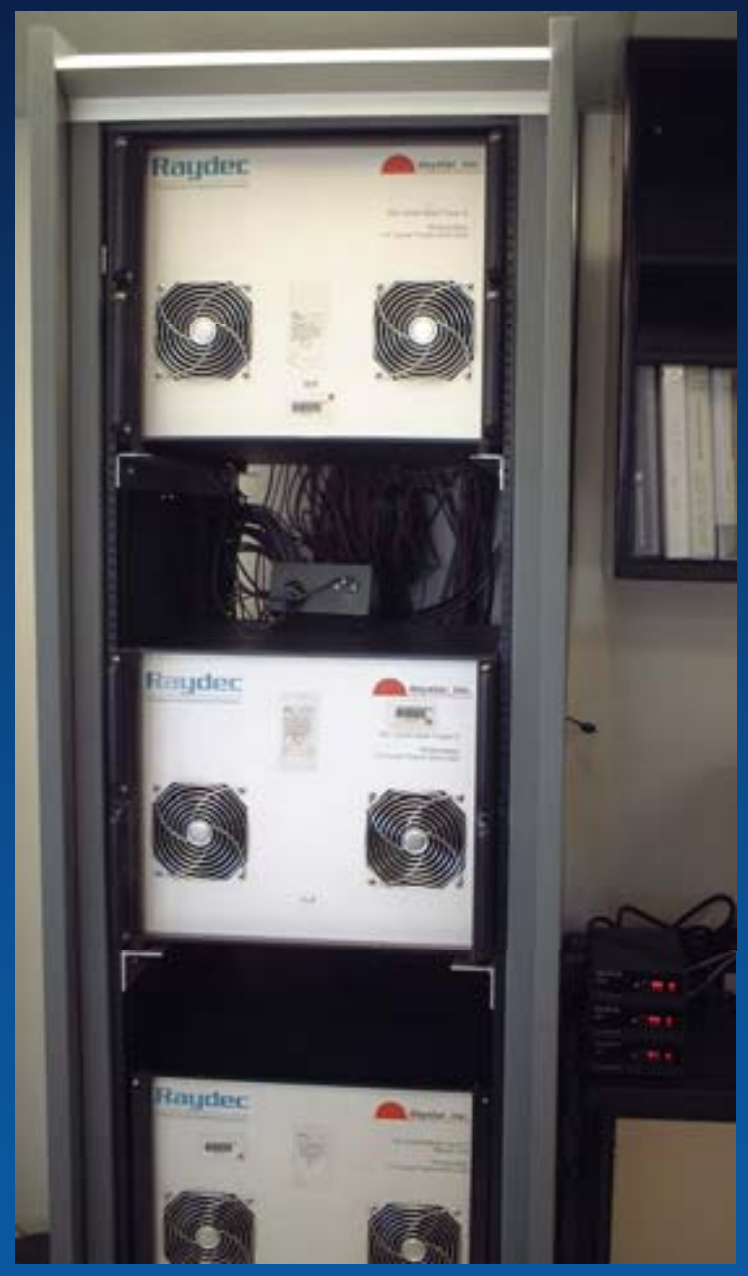




\section{PTC Power Rating Calculations}

- $P_{\max }$ extracted from I-V curves and combined with $\mathrm{E}, \mathrm{T}$, and $\mathrm{s}$

- For $E>800 \mathrm{~W} / \mathrm{m}^{2}, 1$ month of data fit to Performance Test Conditions (PTC)

- Using regression results, power rating @ STC calculated

$$
\begin{aligned}
& P=E\left(a_{1}+a_{2} E+a_{3} T+a_{4} s\right) \\
& \text { PTC: E }=1000 \mathrm{~W} / \mathrm{m}^{2}, \mathrm{~T}=20^{\circ} \mathrm{C}, \mathrm{s}=1 \mathrm{~m} / \mathrm{s}
\end{aligned}
$$




\section{$\mathbf{R}_{\mathrm{D}}$ Determination}

- PTC ratings plotted versus time

- Slope of linear fit gives $R_{D}(-0.35 \% / y r)$

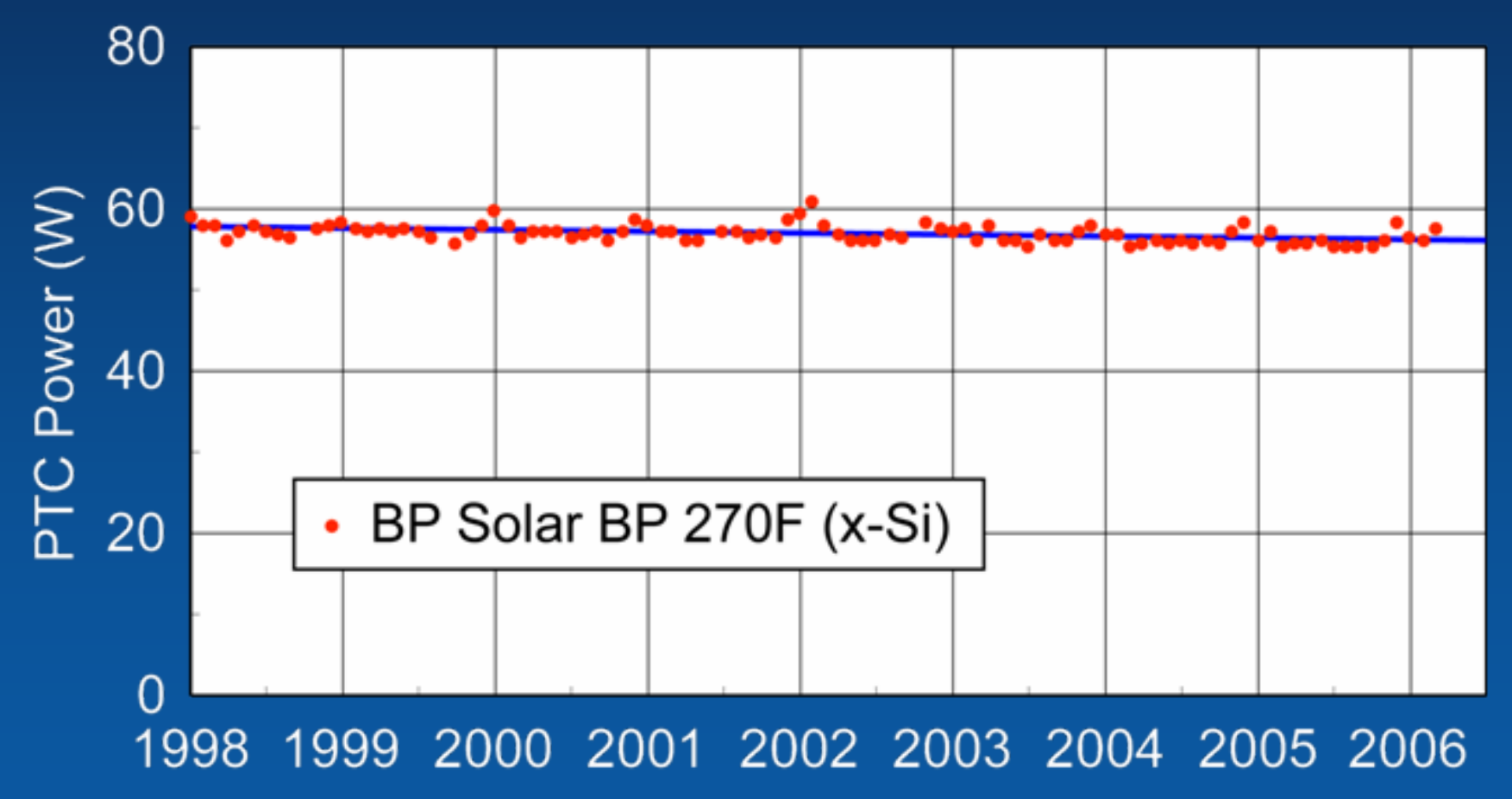




\section{Pitfalls}

- a-Si initial stabilization

- Seasonal variations

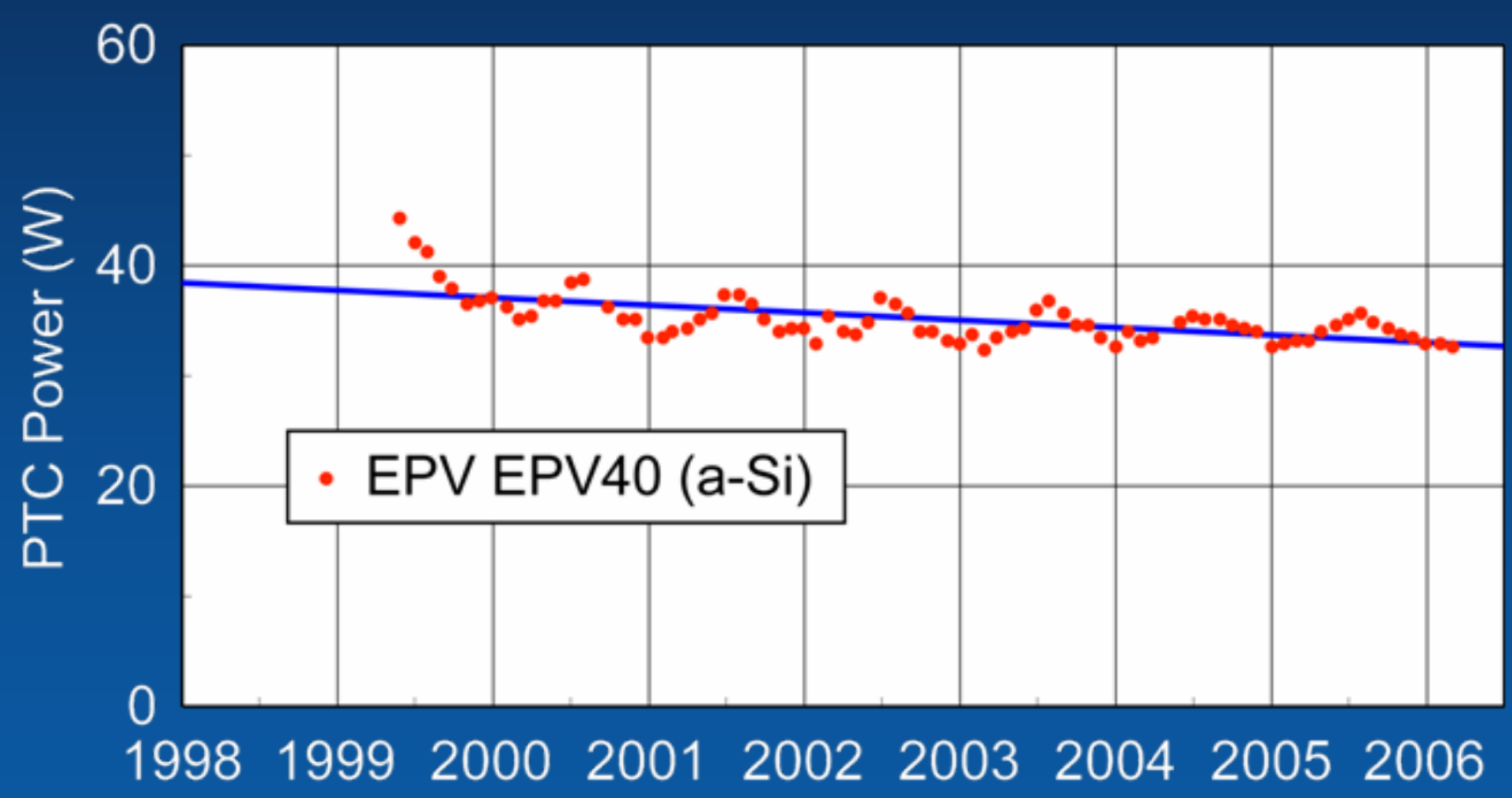




\section{PERT $R_{D}$ Results - Crystalline}

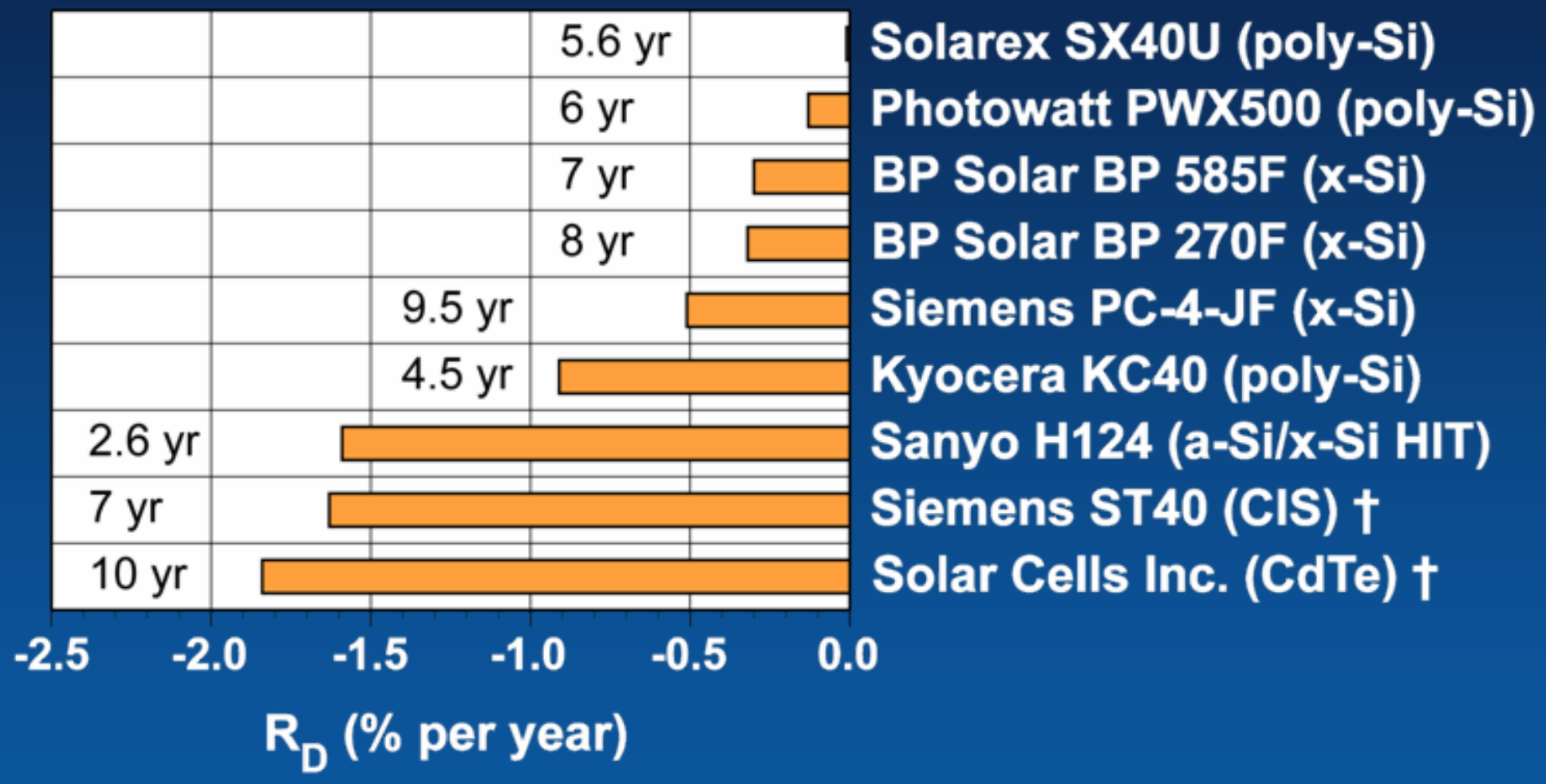




\section{PERT $\boldsymbol{R}_{\mathrm{D}}$ Results - a-Si}

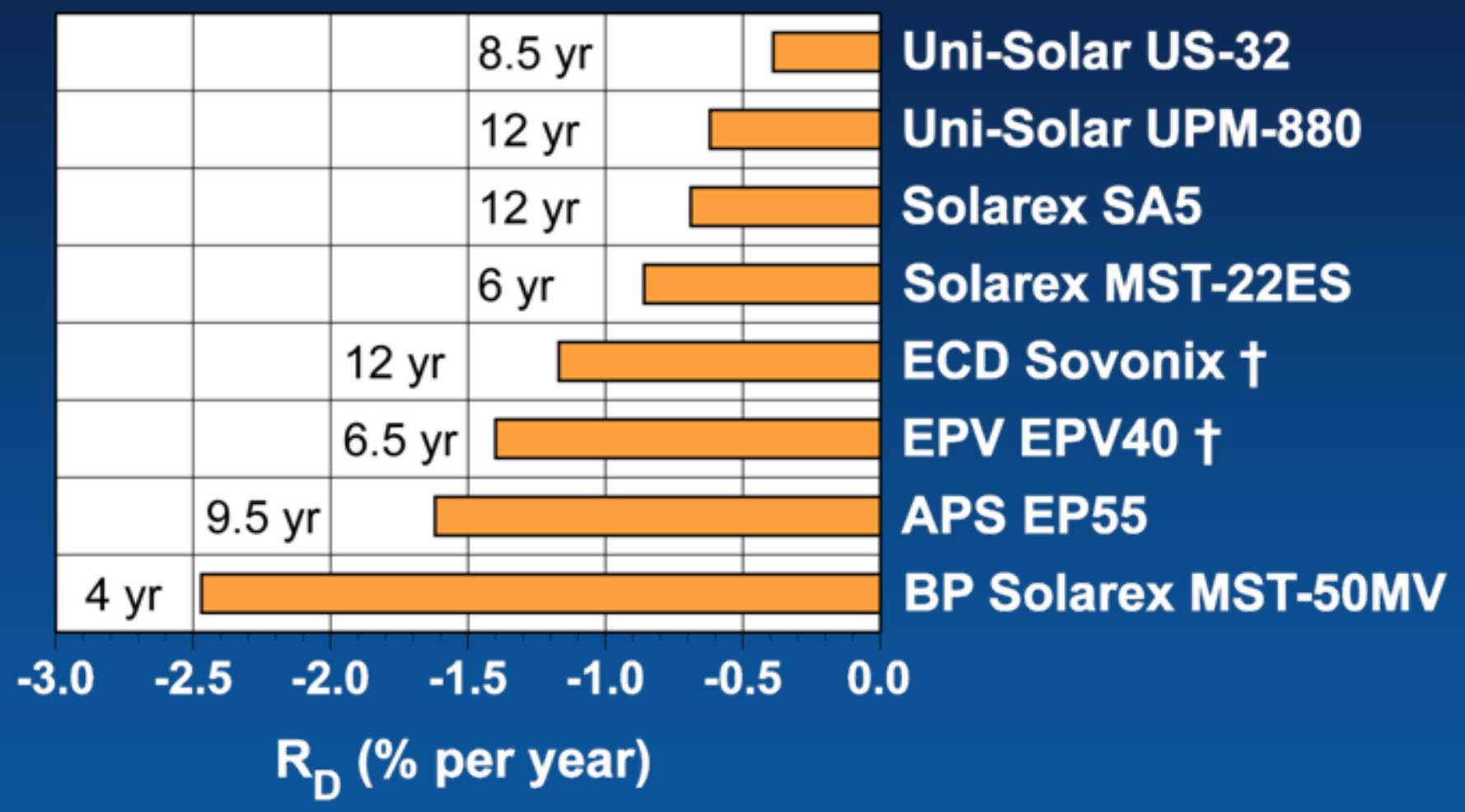

† Non-commercial prototype modules 


\section{Discussion and Conclusions}

- Many Si $R_{D}$ values < 1\%/year

- Some thin-film $R_{D}$ values < $1 \%$ /year

- Recommend $0.5 \%$ per year for Si

- $R_{D}>2 \% / y e a r$ likely indicative of serious module or system problems

- $R_{D}$ values vary over wide range; accurate data should be available for system designers 